\title{
Comparison of the Skin Cancer Quality of Life Impact Tool and the Skin Cancer Index Questionnaire in Measurement of Health-Related Quality of Life and the Effect of Patient Education Brochures in Patients with Actinic Keratosis, Non-melanoma Skin Cancer, and Cutaneous Melanoma
}

\author{
Sam El Abbadi · Laura Susok · Egger Stockfleth · Falk Georges Bechara • Thilo Gambichler • \\ Swetlana Herbrandt · Lisa Goldschmidtböing · Michael Sand
}

Received: December 7, 2020 / Accepted: March 26, 2021 / Published online: April 13, 2021

(C) The Author(s) 2021, corrected publication 2021

\begin{abstract}
Introduction: Few studies have evaluated patient-reported outcome measures and the effect of patient educational materials in German patients with skin cancer. We conducted a prospective study to measure the impact of treatment on health-related quality of life in German patients with skin cancer, identify variables that may contribute to changes in health-related quality of life, and evaluate the effect of providing additional information through a patient education brochure.
\end{abstract}

\section{S. El Abbadi}

Department of Plastic, Reconstructive, Aesthetic and Hand Surgery, University Hospital OWL, Campus

Klinikum Bielefeld Center, Bielefeld, Germany

L. Susok · E. Stockfleth · F. G. Bechara .

T. Gambichler · M. Sand $(\varangle)$

Department of Dermatology, Venereology and

Allergology, Ruhr-University Bochum, Bochum, Germany

e-mail: michael.sand@ruhr-uni-bochum.de

\section{S. Herbrandt · L. Goldschmidtböing}

Department for Statistical Consulting and Analysis, Center for Higher Education, Technical University of Dortmund, Dortmund, Germany

M. Sand

Department of Plastic, Reconstructive and Aesthetic Surgery, St. Josef Hospital, Essen, Germany
Methods: A total of 461 patients diagnosed with actinic keratosis, nonmetastatic nonmelanoma skin cancer, melanoma in situ, or nonmetastatic cutaneous melanoma treated between August 2018 and July 2020 were included. Ninety-seven patients were randomly selected to receive an additional patient education brochure ("Hautkrebs"). The patients completed a Skin Cancer Quality of Life Index Tool $(n=324)$ or a Skin Cancer Index Questionnaire $(n=137) \quad 1$ week after surgical treatment.

Results: Ninety-seven patients were provided an additional patient education brochure. We found statistical correlation between sociodemographic factors (young age and female gender) and the location of the skin cancer (head and face) and impairment of health-related quality of life in patients with skin cancer $(p<0.05)$. Moreover, we found that patients who were presented a brochure showed significantly higher awareness concerning direct sun exposure $(p<0.05)$.

Conclusion: Impaired health-related quality of life is correlated with a patient with skin cancer's age, gender, and the location of the lesion. Physicians should consider these factors in clinical practice and advocate the use of patient education brochures as effective assets in preventing the reoccurrence of skin cancer. 
Keywords: Patient education brochures; Quality of life; SCIQ; SCQOLIT; Skin cancer

\section{Key Summary Points}

The incidence of melanoma (MSCs) and nonmelanoma skin cancer (NMSCs) is increasing in Germany and globally. NMSC is the most prevalent form of cancer among white populations worldwide, and cutaneous melanoma is the most rapidly increasing cancer in white populations recently. Yet only few studies have evaluated patient-reported outcome measures and the effect of patient educational materials in German patients with skin cancer.

We conducted a prospective study to measure the impact of treatment on health-related quality of life (HRQoL) in German patients with skin cancer and identify variables that may contribute to changes in HRQoL. Furthermore, we decided to study the effect of patient information brochures in this context.

We found that impairment of healthrelated quality of life is correlated with a patient with skin cancer's age, gender, and the location of the lesion. Moreover, we found that patients who were presented a patient information brochure showed significantly higher awareness concerning direct sun exposure.

Physicians should therefore consider these factors in clinical practice and advocate the use of patient education brochures as an asset in preventing the reoccurrence of skin cancer. Furthermore, further research is warranted to establish a clinically significant cutoff for the SCIQ and the SCQOLIT to facilitate interpretation of the scores and identification of high-risk patients. In addition, more research is required to establish interventions that may improve HRQoL.

\section{DIGITAL FEATURES}

This article is published with digital features, including a summary slide, to facilitate understanding of the article. To view digital features for this article go to https://doi.org/10.6084/ m9.figshare.14307461.

\section{INTRODUCTION}

The incidence of cutaneous melanoma (CM) and nonmelanoma skin cancer (NMSC) is increasing in Germany and globally [1-3]. NMSC is the most prevalent form of cancer among white populations worldwide $[1,2,4]$, and cutaneous melanoma is the most rapidly increasing cancer in white populations recently [5]. As a result, NMSC is among the top five most expensive malignancies in the USA, creating a financial burden of an estimated US $\$ 1.4$ billion annually [6]. In 2016, approximately 230,000 patients were diagnosed with NMSCs and 23,200 patients were diagnosed with CM in Germany [3].

Health-related quality of life (HRQoL) can be significantly impacted by skin cancer and its treatment [7-14]. Several factors influence HRQoL, including scarring and disfigurement, anxiety, and fears of future skin cancers $[7,15,16]$.

Approximately $30-47 \%$ of patients with CM suffer from significant emotional distress, particularly depression and anxiety symptoms at diagnosis and during treatment $[17,18]$. Several studies suggest that $\mathrm{CM}$ has a greater impact on HRQoL than NMSC and that tumor stage in CM is not associated with impairment of HRQoL $[19,20]$. In terms of treatment, follow-up care, and mortality, low-grade CM resembles NMSC rather than high-grade CM. Therefore, we expect a similar association for HRQoL in both low-grade CM and NMSCs.

Two previous studies in Germany using generic questionnaires showed an impairment of HRQoL in patients with NMSCs. One study included 1184 patients and used the EuroQol 5 dimensions 5 levels (EQ-5D-5L) and found that HRQoL differs between basal cell carcinoma (BCC) and squamous cell carcinoma 
(SCC) on one side and actinic keratosis on the other [21]. Furthermore, they showed lower HRQoL in older patients. Another study in 52 patients that used the Dermatology Life Quality Index found no association between impairment in HRQoL and sociodemographic or clinical factors [22]. These findings differ from the results of several studies outside of Germany using disease-specific questionnaires in patients with NMSCs [23]. Therefore, we conducted a cross-sectional pilot study to measure the impact of treatment on HRQoL, identify variables that may contribute to changes in HRQoL, and test the applicability of two of the most common patient-reported outcome measures (PROMs) in a German sample of patients with skin cancer.

One such variable may be patient education and knowledge concerning skin cancer. Patient education enables patients to understand their disease and supports self-guided decision-making and coping [24, 25]. However, low levels of health literacy have been associated with poor health outcomes [26]. Thus, patient education may have a direct impact on HRQoL. Several studies have revealed an alarmingly low level of knowledge and disease-understanding among patients with skin cancer before and after treatment [27-30]. Patient education brochures are highly accepted in older patients and among the more heavily used sources of information for cancer patients [31-33].

Furthermore, information brochures have supported favorable changes in lifestyle in older patients with chronic impairments and patients with chronic diseases [34, 35]. Therefore, we implemented patient education brochures as an intervention and studied their effect on HRQoL.

\section{METHODS}

The Research Ethics Committee of the Nordrhein Medical Council in Duesseldorf (Germany) approved this study. The study was performed in accordance with the Declaration of Helsinki 1964 and its later amendments. All participants provided informed consent to participate in the study. We conducted a crosssection study from August 2018 to July 2020 at the Departments for Plastic and Reconstructive Surgery in both Bielefeld and Essen. All patients who presented to our clinic were asked to participate. Patients with other additional malignancies, life-threatening illnesses, or those unable to consent were excluded. We included 461 patients referred for surgical treatment of nonmetastatic NMSC, actinic keratosis, CM in situ, or CM. Patients who were referred more than once during treatment of multiple skin cancers were enrolled only for their first cancer.

Ninety-seven patients were randomly selected to receive an additional patient education brochure [36] designed and published by the German Cancer Aid. The brochure consists of 12 chapters and 120 pages and provides information on early detection measures, causes for skin cancer, diagnosis, treatment, clinical studies, rehabilitation, and aftercare. Moreover, it contains a collection of contacts for further information and psychological and financial support for patients with cancer. The brochure is not commercially sponsored and is accepted by the German Association for Dermatologic Oncology (ADO) [36]. German Cancer Aid is an independent, nonprofit foundation dedicated to fighting cancer. The blue guides ("die blauen Ratgeber") are informational booklets developed and published for various cancer types and are provided complimentary to patients. As part of their clinical care patients were asked to complete the Skin Cancer Quality of Life Impact Tool (SCQOLIT) or the Skin Cancer Index Questionnaire (SCIQ) at their 1-week follow-up. Patients who received postoperative care elsewhere were given an SCIQ or SCQOLIT to complete after 1 week with instruction to send the questionnaire back by mail.

Questionnaires that were not sent back within 3 weeks after surgery were not included in the study. The SCIQ and the SCQOLIT are skin cancer-specific PROMs. The SCIQ was validated for patients with NMSCs in the face and consisted of 15 items using a 5-point Likert scale. Patients choose from five response choices, ranging from "not at all" (5) to "very much" (1). SCIQ responses are summarized in three subscales, addressing three domains: emotional, social, and appearance. Scores vary from 5 (least effect on quality of life) to 1 (most effect on 
quality of life). After adding up each subscale score, the scores are standardized on a scale from 0 to 100 .

Patients were asked to respond specifically about the effects of their skin cancer and its treatment for the last week after surgery, and higher scores indicate better HRQoL.

To evaluate the effect of receiving a patient education brochure, we investigated total scores, scores for the subscales, and scores for items $1-4$ and 6 of the SCIQ. These items explicitly ask about fears concerning the cause and progress of skin cancer.

The SCQOLIT was validated for nonmetastatic CM and nonmetastatic NMSC and consisted of 10 items using a 4-point Likert scale. Patients choose from four response choices that range from "not at all" (0) to "very much" (3). Scores vary from 3 (highest effect on health-related QoL) to 0 (least effect). Patients were asked to respond specifically about their skin cancer effects and its treatment for the last week after surgery. In contrast to the SCIQ, lower SCQOLIT scores indicate better HRQoL (range 0-30). We evaluated the effect of receiving a patient education brochure by investigating total scores and scores for items 2 and 3 of the SCQOLIT. Item 2 of the SCQOLIT asks about the patient's need for more information on recognizing and preventing skin cancer; item 3 asks how much the patient has worried about covering up the skin and keeping out of the sun for the last week. Total scores (SCIQ and SCQOLIT) were only calculated for complete questionnaires.

The questionnaires were translated into German. The Cronbach's $\alpha$ coefficients were computed for both questionnaires after the translation. Both demonstrated excellent internal consistency (SCQOLIT $\alpha=0.878$, SCIQ $\alpha=0.921$ ), indicating no relevant distortion from the translation.

\section{Statistical Analysis}

The association between patient variables and scores for both questionnaires was determined using the $t$ test, analysis of variance, and post hoc tests. Pearson's chi-squared test and Fisher's
Table 1 Patient demographic data

\begin{tabular}{ll}
\hline Demographic variables & Value \\
\hline Gender $(n, \%)$ & $222(48.2 \%)$ \\
Female & $239(51.8 \%)$ \\
Male & \\
Age (years) & $73.16(12)$ \\
Mean (SD) & $23-102$ \\
\hline
\end{tabular}

$S D$ standard deviation

exact test were used to determine the correlation between isolated items and patient variables. Cramér's $V$ was used to determine the correlation between patient variables on a nominal scale. We used the $t$ test to compare our mean scores with the mean scores reported in the literature. Two-tailed $p$ values of 0.05 or less were considered statistically significant. All statistical analyses were calculated using IBM SPSS Statistics for Windows, Version 26.0 (Armonk, NY: IBM Corp.).

\section{RESULTS}

A total of 461 patients were included. The mean patient age was 73.2 years (range 23-102). A slight majority were male (239 men, 51.8\%; 222 women, $48.2 \%$ ), and most patients were affected by basal cell cancers $(65.5 \%, n=302)$. The most common site of appearance was the head and face $(91.3 \%, n=375)$. The most frequently affected region in the face was the nose and cheek. Eighty-six patients (18.7\%) presented more than one lesion. Table 1 presents patient demographic data, and Table 2 presents lesion data such as size, location, and thickness. The scores for the SCIQ and SCQOLIT are shown in Table 3.

Analysis of the SCQOLIT showed significantly higher scores for the face and head tumors $(n=273,84 \%)$ than tumors of the trunk and extremities $(n=52,16 \% ; p<0.05)$. 
Table 2 Lesion data

\begin{tabular}{|c|c|}
\hline Lesion data & $N(\%)$ \\
\hline \multicolumn{2}{|l|}{ Type } \\
\hline $\mathrm{BCC}$ & $302(65.5 \%)$ \\
\hline $\mathrm{AK}$ & $73(15.8 \%)$ \\
\hline SCC & $55(11.9 \%)$ \\
\hline $\mathrm{CM}$ & $22(4.8 \%)$ \\
\hline Melanoma in situ & $9(2.0 \%)$ \\
\hline \multicolumn{2}{|l|}{ Quantity } \\
\hline More than 1 lesion & $86(18.7 \%)$ \\
\hline \multicolumn{2}{|l|}{ Location } \\
\hline Scalp & $53(11.5 \%)$ \\
\hline Forehead & $64(13.9 \%)$ \\
\hline Orbital region & $43(9.3 \%)$ \\
\hline Nose & $94(20.4 \%)$ \\
\hline Cheek & $73(15.8 \%)$ \\
\hline Ears & $28(6.1 \%)$ \\
\hline Lips & $16(3.5 \%)$ \\
\hline Chin & $4(0.9 \%)$ \\
\hline Extremities & $44(9.5 \%)$ \\
\hline Trunk & $42(9.1 \%)$ \\
\hline \multicolumn{2}{|l|}{ Thickness } \\
\hline Mean (SD) & $1.86 \mathrm{~mm}(1.66)$ \\
\hline Range & $0-15 \mathrm{~mm}$ \\
\hline \multicolumn{2}{|l|}{ Expansion } \\
\hline Mean (SD) & $10.56 \mathrm{~mm}(7.89)$ \\
\hline Range & $1.1-60 \mathrm{~mm}$ \\
\hline
\end{tabular}

$B C C$ basal cell carcinoma, $A K$ actinic keratosis, $S C C$ squamous cell carcinoma, $C M$ cutaneous melanoma, $S D$ standard deviation
Table 3 Questionnaire scores

\begin{tabular}{lll}
\hline Questionnaire score & Mean (SD) & Number \\
\hline SCQOLIT & $14.55(7.72)$ & 325 \\
Total score & & \\
SCIQ & $53.55(13.29)$ & 113 \\
Nonstandard total score & $67.11(22.24)$ & 113 \\
Standard total score & $51.77(27.29)$ & 119 \\
Emotional subscale & $75.59(21.27)$ & 119 \\
Social subscale & $72.31(31.51)$ & 119 \\
Appearance subscale & Quality of Life Impact Tool, \\
\hline SCQOLIT Skin Cancer &
\end{tabular}

No significant correlation was apparent between receiving a brochure and total scores and scores for item 2 of the SCQOLIT $(p>0.05)$.

Analysis of scores for item 3 of the SCQOLIT showed significant differences between patients who received an education brochure $(n=59$, $18.1 \%)$ and patients who did not receive a brochure $(n=266,81.8 \%$; $p<0.05)$.

Analysis of the SCIQ showed a significant correlation between age and scores (total score, emotional subscale, appearance subscale) for all tumors $(p<0.05)$.

Moreover, significant differences between men $(n=55,51.8 \%)$ and women $(n=51$, $48.1 \%)$ were shown for NMSCs $(p<0.05)$. No significant correlation was apparent between total scores or subscale scores and receiving an information brochure $(p>0.05)$. Furthermore, there was no correlation between the scores of specific items (items 1-4 and 6) and receiving an information brochure.

\section{DISCUSSION}

As a result of an improved understanding of the biology of nonmelanoma skin cancers, innovative treatment options, including the Hedgehog pathway and immunotherapeutic agents, have been developed in recent years. However, surgery remains the primary option for NMSCs [37]. 
NMSC has a better survival prognosis than most cancers, but patients with NMSC still must deal with the distress and emotional disturbances attributed to a cancer diagnosis [16]. Moreover, they are often burdened by highly visible disfigurement [16]. Distress and concerns are comprehensible since BCC is most found in the face with the highest local incidence on the nose [2]. The highest body site-specific incidence rates include solely exposed and visible areas such as the lip, orbit, nasolabial, ear, nose, cheek, and the dorsum of the hands [38]. In comparison to Choi et al. [39] who investigated the distribution of NMSC concerning the aesthetic units of the face, we found similar results with the highest occurrence of BCC on the nose and central face and, for SCC, on the cheeks. Therefore, it is not surprising that HRQoL can be significantly impacted by skin cancer and its surgical treatment [7-14].

We undertook this pilot study to measure the impact of skin cancer and its treatment on HRQoL in German patients with skin cancer, identify variables that may contribute to changes in HRQoL, and evaluate the effect of providing additional information through a patient education brochure. Furthermore, we aimed to test the applicability of two of the most used PROMs in a German sample. To our knowledge, this is the first study in German patients with skin cancer using disease-specific questionnaires for measurement of HRQoL.

Statistical analysis of the scores for the SCQOLIT demonstrated a significantly higher impairment of HRQoL for tumors of the face and head compared with tumors of the trunk and extremities $(p \leq 0.05)$. Burdon-Jones et al. reported in 2010 that $45 \%$ of patients with NMSCs were concerned about the possibility of scarring or disfigurement, particularly on the face [7].

Lee et al. [40] undertook 15 in-depth interviews in 2016 with patients who underwent surgery to remove skin cancer in the face as a basis for the development of a new PROM. They found that the aesthetic outcome after surgery had significant implications for the patients' psychological and social well-being. Borah et al. [41] demonstrated how crucial the coherence between well-being and facial appearance is in
2009 by conducting a study on 210 people using the Gamble Chance of Death Questionnaire. They found that half of the adults would risk at least a $7 \%$ chance of death to obtain a normal physical appearance, and more than $13 \%$ of adults would accept even a $30-45 \%$ risk of death to obtain a normal face [41]. Moreover, the face was ranked as the most important area for reconstruction after trauma and functional reconstruction [41]. The authors concluded that a normal appearance is a primary function of the face. A review conducted in 2015 emphasized the importance of facial appearance as well, and they reasoned that, particularly for patients with skin cancer, an understanding of this matter might improve the informed consent process and preoperative and postoperative counseling [42].

Analysis of the SCIQ showed a significant correlation between old age and greater HRQoL (total score, emotional subscale, and appearance subscale) for all tumors $(p \leq 0.05)$. Moreover, we found significantly lower HRQoL in women with NMSC $(p \leq 0.05)$. These findings are congruent with Rhee et al.'s results [13], which demonstrated lower HRQoL for women and patients younger than age 50 as part of the validation of the SCIQ for NMSC.

Zhang et al. [11] conducted a study in 2018 on 727 patients with NMSC and CM. They reported significantly lower HRQoL for women and patients younger than 65 years using the SCIQ compared to patients older than 65 .

Sobanko et al. [43] examined 136 patients using the SCIQ and the Hospital Anxiety and Depression Scale, showing likewise lower HRQoL for younger patients with skin cancer and women. A Spanish study conducted in 2016 using the SCIQ in 88 patients found lower HRQoL associated with younger age and female sex, as well [44]. Vaidya et al. [45] discovered that lower postoperative facial appearance and scar satisfaction scores were associated with female sex, younger age, and location on the nose. Lower HRQoL in women might be related to cultural concerns about their health and appearance [46]. Furthermore, according to Rhee et al. [13], previous research suggests that women may experience more difficulty adapting to cervicofacial cancers because they may 
potentially value facial attractiveness more than men. One explanation for the association of a patient's age and HRQoL could be rooted in the trend that as patients age, their value in physical appearance tends to decline.

The most well-recognized exogenous factor implicated in the pathogenesis of NMSC is ultraviolet radiation (UVR) [47]. Having a history of skin cancer is a risk factor for developing a new skin cancer lesion [48]. Therefore, avoidance of direct sun exposure is of utmost importance as a secondary prophylactic measure. We found that patients who received an informational brochure showed a significantly higher awareness concerning direct sun exposure $(p \leq 0.05)$.

Falk et al. [48] investigated sun exposure habits among patients with a history of skin cancer and found that those in the skin cancer group reported a consistently higher level of sun avoidance than those in the control group. Also, several studies reported that the use of sun-protective behaviors increased after treatment $[49,50]$. de Blacam et al. [30] showed that a majority of patients with previously treated BCC $(71.2 \%)$ were aware of the harmful effects of UVR, and most (more than 70\%) employed some sort of sun protection, including the avoidance of midday sun, staying in the shade, and wearing a hat or sunscreen. This implies that up to $30 \%$ of patients with NMSCs in their history are insufficiently informed and do not perform sun protection measures in a recommended manner. Patient information brochures may be an asset in educating patients about secondary prevention and initiating favorable lifestyle changes.

Also, de Blacam et al. [30] found that only $28.8 \%$ of respondents understood the excised lesion was a BCC and that there was a significant chance of developing another similar lesion over the next few years [30]. In this context, patient education brochures may help patients understand their diagnosis and help prevent delayed clinical presentation in cases of recurrence.

Our study showed no relevant association between Breslow thickness and HRQoL. This was expected given that all the CM included in the study presented a Breslow thickness less than $2 \mathrm{~mm}$, necessitating narrow excision margins of $1 \mathrm{~cm}$ according to the latest European Society for Medical Oncology (ESMO) guidelines [51]. Most of the CM were low-risk melanoma with a thickness less than $0.8 \mathrm{~mm}$ with only three exceptions.

Furthermore, we found no association between size and HRQoL. Since most NMSCs grow relatively slowly, the magnitude of a skin cancer lesion itself might be an indicator for low impairment in a specific individual; there might be a negative correlation between the size of slow-growing skin cancer and impairment of HRQOL.

In the measurement of HRQoL, we found no statistical difference between NMSCs, actinic keratosis, CM, and $\mathrm{CM}$ in situ. As previously stated, we only included low-risk melanoma into this study. Therefore, the expected mortality in all patients was similarly low. Furthermore, the primary surgical treatment and the follow-up treatment are similar, too. Because of our study's cross-sectional character, we did not recognize differences in the recovery of HRQoL. A recent study performed on 247 patients, including actinic keratosis, NMSC, and CM utilizing the EQ-5D-5L questionnaire showed impairment of HRQoL for all individuals immediately after surgery with decreased HRQoL in CM even 1 year after surgery [52].

Since low-risk melanoma (stage I) necessitates safety margins of only $1 \mathrm{~cm}$, and most of the CMs are located on the trunk, primary closure is often possible, eliminating the need for skin grafting or flap repair. However, the impact of wider excisional margins on HRQoL is not clearly recognizable. Bergenmar et al. [53] compared narrow $(2 \mathrm{~cm})$ and wide $(4 \mathrm{~cm})$ margins for excision of melanoma and found no difference in QoL after surgery. Newton-Bishop et al. [54] conducted a postal questionnaire study, including 426 patients with high-risk melanomas. They compared patients with a $3-\mathrm{cm}$ excision margin with patients that had a 1 -cm excision margin [54]. After 1 month, the $3-\mathrm{cm}$ excision group had significantly poorer mental and physical functioning [54]. However, these differences disappeared within 6 months except for a persisting poor scar perception in the $3-\mathrm{cm}$ excision group [55]. A 3-cm excision 
margin and younger age, female gender, and poor physical and mental health post surgery were associated with poor scar perception [54]. In this context, according to the latest ESMO guidelines, excision margins wider than $2 \mathrm{~cm}$ are not recommended [51].

Finally, our median scores for SCIQ (range 0-100; high scores indicate high HRQoL) and SCQOLIT (range 0-30; low scores indicate high HRQoL) 1 week after surgery compared to Anglo-Saxon authors indicated a significantly lower HRQoL in our patients [12, 13, 19, 55].

Wali et al. [55] reported a median score for the SCQOLIT in British patients of 5.33 (SD 5.32) at baseline. Burdon-Jones [19] reported a median score for melanoma of 10 (SD 12) and for NMSC of 4 (SD 5) at baseline [19]. However, we established a median score of 14.4 for melanoma (SD 7.15) and 14.56 (SD 7.7) for NMSCs.

Sanchez et al. [12] reported unstandardized total scores for the SCIQ (15-75, high scores indicating high HRQoL) with a mean score of 65.07 for Mohs surgery after 1-2 weeks and 62.82 at $1-2$ weeks after conventional surgery. Likewise, our median scores (MW 53.55; SD 13.489) are significantly lower, indicating lower HRQoL. We compared our results to the findings of Rhee et al. as well [13]. We found significant differences in total scores, emotional and social subscales. No significant differences were found concerning the appearance subscale.

Interestingly, de Troya-Martín et al. [44] reported findings that were similar to ours: female gender and young age were associated with low HRQoL in measurement with the Spanish version of the SCIQ (range 0-100) [43]. One week after surgery, the mean score was 63.8 (SD 20.2). The authors stated that, unlike other studies conducted in Anglo-Saxon countries, their emotional subscale values were remarkably lower than those for the social appearance component for all time points and all groups of patients [43]. These findings are congruent with our results, with the greatest impairment in the emotional subscale and a mean score of 67.11 (SD 22.24) after 1 week. These findings might be due to cultural differences between continental Europe and Anglo-Saxon nations or different medical systems. However, it must be noted that three items were removed in the validation process of the Spanish version of the SCIQ [56]. As the Spanish version retains the standardized overall score of the original scale, the values obtained with the two instruments (0-100) are equivalent from a quantitative viewpoint [56]. Nevertheless, the elimination of three items and the Spanish version's reduced dimensions could complicate comparing it with the original SCI [56].

The SCQOLIT is a short PROM with only 10 items, making it a good candidate for incorporation into busy skin cancer clinics with a low administrative burden $[19,55]$. The SCQOLIT was validated for NMSC and CM. Therefore, it is not specific to NMSC, and it does not address detailed cosmetic concerns [56]. The SCQOLIT elicits predominantly psychosocial worries, with only question 8 addressing a physical concern [19]. This might be why the SCQOLIT fails to identify differences in HRQoL between men and women. The SCI has been specifically validated in patients with NMSCs treated with Mohs micrographic surgery [15].

The SCI showed acceptable statistical characteristics [57], but clinicians in Germany do not commonly perform Mohs micrographic surgery. Furthermore, only patients with NMSCs located on the head and neck were included in the validation process [15]. Therefore, the sample of patients featured in the SCI validation is not representative of all patients with NMSC [57].

Interestingly, both questionnaires showed correlations between HRQoL and different variables (age, gender, and skin cancer location). This highlights the difficulty in comparing the results of PROMs in the measurement of HRQoL, even for the same illness.

Our study was limited by its cross-sectional design, incomplete patient survey data, and missing power analysis before our testing. Furthermore, the introduction of an English PROM for a German cohort necessitates a forward-backward translation and a subsequent psychometric validation. This validation was not performed as part of this pilot study. Therefore, the implications of our findings are constrained. A prospective study to validate a 
German version of the SCQOLIT is currently in preparation.

\section{CONCLUSION}

Sociodemographic factors (young age and female gender) and the location of the skin cancer (head and neck) may be associated with impairment of HRQoL in patients with skin cancer in Germany.

Clinicians should consider these risk factors to identify patients who may benefit from additional counseling. Moreover, patient education brochures may be an asset in educating patients on secondary prevention and initiating favorable lifestyle changes.

Furthermore, further research is warranted to establish a clinically significant cutoff for the SCIQ and the SCQOLIT to facilitate interpretation of the scores and identification of high-risk patients. In addition, more research is required to establish interventions that may improve HRQOL.

\section{ACKNOWLEDGEMENTS}

The authors would like to thank patients and staff at the St. Josef's Hospital Essen and Klinikum Bielefeld Center for their time and cooperation in this study.

Funding. No funding or sponsorship was received for this study or publication of this article.

Authorship. All named authors meet the International Committee of Medical Journal Editors (ICMJE) criteria for authorship for this article, take responsibility for the integrity of the work as a whole, and have given their approval for this version to be published.

Disclosures. No authors involved in this manuscript's production have any commercial associations that might create or pose a conflict of interest with information presented herein.
Compliance with Ethics Guidelines. The Research Ethics Committee of the Nordrhein Medical Council in Duesseldorf (Germany) approved this study. The study was performed in accordance with the Declaration of Helsinki 1964 and its later amendments. All participants provided informed consent to participate in the study.

Data Availability. The datasets generated during and/or analyzed during the current study are available from the corresponding author on reasonable request.

Open Access. This article is licensed under a Creative Commons Attribution-NonCommercial 4.0 International License, which permits any non-commercial use, sharing, adaptation, distribution and reproduction in any medium or format, as long as you give appropriate credit to the original author(s) and the source, provide a link to the Creative Commons licence, and indicate if changes were made. The images or other third party material in this article are included in the article's Creative Commons licence, unless indicated otherwise in a credit line to the material. If material is not included in the article's Creative Commons licence and your intended use is not permitted by statutory regulation or exceeds the permitted use, you will need to obtain permission directly from the copyright holder. To view a copy of this licence, visit http://creativecommons.org/licenses/by$\mathrm{nc} / 4.0 /$.

\section{REFERENCES}

1. Lomas A, Leonardi-Bee J, Bath-Hextall F. A systematic review of worldwide incidence of nonmelanoma skin cancer. Br J Dermatol. 2012;166(5): 1069-80.

2. Leiter U, Garbe C. Epidemiology of melanoma and nonmelanoma skin cancer-the role of sunlight. AdvExp Med Biol. 2008;624:89-103.

3. Krebs in Deutschland für 2015/2016. Korrigierte 12 . Ausgabe. Robert Koch-Institut (Hrsg) und die Gesellschaft der epidemiologischen Krebsregister in Deutschland e.V. (Hrsg). Berlin; 2019. 
4. Kim RH, Armstrong AW. Nonmelanoma skin cancer. Dermatol Clin. 2012;30(1):125-39, ix.

5. Leiter U, Eigentler T, Garbe C. Epidemiology of skin cancer. Adv Exp Med Biol. 2014;810:120-40.

6. Housman TS, Feldman SR, Williford PM, et al. Skin cancer is among the most costly of all cancers to treat for the Medicare population. J Am Acad Dermatol. 2003;48(3):425-9.

7. Burdon-Jones D, Thomas P, Baker R. Quality of life issues in nonmetastatic skin cancer. Br J Dermatol. 2010;162:147-51.

8. Cornish D, Holterhues C, van de Poll-Franse LV, Coebergh JW, Nijsten T. A systematic review of health-related quality of life in cutaneous melanoma. Ann Oncol. 2009;20(Suppl 6):vi51-8.

9. Holterhues C, Cornish D, van de Poll-Franse LV, et al. Impact of melanoma on patients' lives among 562 survivors: a Dutch population-based study. Arch Dermatol. 2011;147(2):177-85.

10. Gaulin C, Sebaratnam DF, Fernández-Peñas P. Quality of life in non-melanoma skin cancer. Australas J Dermatol. 2015;56(1):70-6.

11. Zhang J, Miller CJ, O’Malley V, Etzkorn JR, Shin TM, Sobanko JF. Patient quality of life fluctuates before and after Mohs micrographic surgery: a longitudinal assessment of the patient experience. J Am Acad Dermatol. 2018;78(6):1060-7.

12. Sanchez N, Griggs J, Nanda S, et al. The Skin Cancer Index: quality-of-life outcomes of treatments for nonmelanoma skin cancer. J Dermatol Treat. 2020;31(5):491-3.

13. Rhee JS, Matthews BA, Neuburg M, Logan BR, Burzynski $M$, Nattinger $\mathrm{AB}$. The skin cancer index: clinical responsiveness and predictors of quality of life. Laryngoscope. 2007;117(3):399-405.

14. Chren MM, Sahay AP, Bertenthal DS, Sen S, Landefeld CS. Quality-of-life outcomes of treatments for cutaneous 274 basal cell carcinoma and squamous cell carcinoma. J Investig Dermatol. 2007;127:1351-7.

15. Rhee JS, Matthews BA, Neuburg M, Burzynski M, Nattinger AB. Creation of a quality of life instrument for nonmelanoma skin cancer patients. Laryngoscope. 2005;115:1178-85.

16. Radiotis G, Roberts N, Czajkowska Z, Khanna M, Körner A. Nonmelanoma skin cancer: diseasespecific quality-of-life concerns and distress. Oncol Nurs Forum. 2014;41(1):57-65.
17. Kasparian NA, McLoone JK, Butow PN. Psychological responses and coping strategies among patients with malignant melanoma. Arch Dermatol. 2009;145(12):1415-27.

18. Loquai C, Scheurich V, Syring N, et al. Screening for distress in routine oncological care-a survey in 520 melanoma patients. PLoS One. 2013;8(7):e66800.

19. Burden-Jones D, Gibbons K. The Skin Cancer Quality of Life Impact Tool (SCQOLIT): a validated health-related quality of life questionnaire for nonmetastatic skin cancers. J Eur Acad Dermatol Venereol. 2013;27:1109-13.

20. Krajewski C, Benson S, Elsenbruch S, Schadendorf D, Livingstone E. Predictors of quality of life in melanoma patients 4 years after diagnosis: results of a nationwide cohort study in Germany. J Psychosoc Oncol. 2018;36(6):734-53.

21. Philipp-Dormston WG, Müller K, Novak B, et al. Patient-reported health outcomes in patients with nonmelanoma skin cancer and actinic keratosis: results from a large-scale observational study analysing effects of diagnoses and disease progression. J Eur Acad Dermatol Venereol. 2018;32(7):1138-46.

22. Steinbauer J, Koller M, Kohl E, Karrer S, Landthaler M, Szeimies RM. Quality of life in health care of non-melanoma skin cancer-results of a pilot study. J Dtsch Dermatol Ges. 2011;9(2):129-35.

23. Mori S, Lee EH. Beyond the physician's perspective: a review of patient-reported outcomes in dermatologic surgery and cosmetic dermatology. Int J Womens Dermatol. 2018;5(1):21-6.

24. Cassileth BR, Lusk EJ, Bodenheimer BJ, Farber JM, Jochimsen P, Morrin-Taylor B. Chemotherapeutic toxicity-the relationship between patients' pretreatment expectations and posttreatment results. Am J Clin Oncol. 1985;8(5):419-25.

25. Iconomou G, Viha A, Koutras A, Vagenakis AG, Kalofonos HP. Information needs and awareness of diagnosis in patients with cancer receiving chemotherapy: a report from Greece. Palliat Med. 2002;16(4):315-21.

26. Mõttus R, Johnson W, Murray C, Wolf MS, Starr JM, Deary IJ. Towards understanding the links between health literacy and physical health. Health Psychol. 2014;33(2):164-73. https://doi.org/10.1037/ a0031439.

27. Bath-Hextall F, Jenkinson C, Kumar A, et al. Longitudinal, mixed method study to look at the experiences and knowledge of non melanoma skin cancer from diagnosis to one year. BMC Dermatol. 2013;13:13. 
28. Renzi C, Mastroeni S, Mannooranparampil TJ, et al. Delay in diagnosis and treatment of squamous cell carcinoma of the skin. Acta Derm Venereol. 2010;90(6):595-601.

29. Yancovitz M, Rizzo C, Julian P, Oliveria SA, Becker D. Patient perceptions of nonmelanoma skin cancer before diagnosis. Dermatol Surg. 2013;39: 1496-501.

30. de Blacam C, Dermott CM, Sugrue C, Kilmartin D, Kelly J. Patient awareness and sun protection behaviour following excision of basal cell carcinoma. Surgeon. 2017;15(1):12-7.

31. Lins S, Icks A, Meyer G. Understanding, comprehensibility and acceptance of an evidence-based consumer information brochure on fall prevention in old age: a focus group study. BMC Geriatr. 2011;11:26.

32. Ankem K. Use of information sources by cancer patients: results of a systematic review of the research literature. Inf Res. 2006;11(3):paper 254.

33. Steeb T, Wessely A, Mastnik S, et al. Patient attitudes and their awareness towards skin cancer-related apps: cross-sectional survey. JMIR Mhealth Uhealth. 2019;7(7):e13844.

34. Wallhagen MI, Strawbridge WJ. Hearing loss education for older adults in primary care clinics: benefits of a concise educational brochure. Geriatr Nurs. 2017;38(6):527-30.

35. Plow M, Bethoux F, Mai K, Marcus B. A formative evaluation of customized pamphlets to promote physical activity and symptom self-management in women with multiple sclerosis. Health Educ Res. 2014;29(5):883-96.

36. Stiftung Deutsche Krebshilfe, DKG Krebsgesellschaft. Die blauen Ratgeber 05 Haut Krebs: Antworten. Hilfen. Perspektiven. Bonn: Stiftung Deutsche Krebshilfe; 2018. https://www.krebshilfe. de/infomaterial/Blaue_Ratgeber/Hautkrebs_

BlaueRatgeber_DeutscheKrebshilfe.pdf. Accessed 18 Mar 2021.

37. Cives M, Mannavola F, Lospalluti L, et al. Nonmelanoma skin cancers: biological and clinical features. Int J Mol Sci. 2020;21(15):5394.

38. Buettner PG, Raasch BA. Incidence rates of skin cancer in Townsville, Australia [published correction appears in Int J Cancer 2001 Jul 15;93(2): 302-3]. Int J Cancer. 1998;78(5):587-93.

39. Choi JH, Kim YJ, Kim H, Nam SH, Choi YW. Distribution of basal cell carcinoma and squamous cell carcinoma by facial esthetic unit. Arch Plast Surg. 2013;40(4):387-91.
40. Lee EH, Klassen AF, Lawson JL, Cano SJ, Scott AM, Pusic AL. Patient experiences and outcomes following facial skin cancer surgery: a qualitative study. Australas J Dermatol. 2016;57(3):e100-4.

41. Borah GL, Rankin MK. Appearance is a function of the face. Plast Reconstr Surg. 2010;125(3):873-8.

42. Sobanko JF, Sarwer DB, Zvargulis Z, Miller CJ. Importance of physical appearance in patients with skin cancer. Dermatol Surg. 2015;41(2):183-8.

43. Sobanko JF, Zhang J, Margolis DJ, et al. Patient-reported quality of life and psychosocial health prior to skin cancer treatment-a cross-sectional study. J Am Acad Dermatol. 2016;75(1):217-218.e2.

44. de Troya-Martín M, Rivas-Ruiz F, Blázquez-Sánchez $\mathrm{N}$, et al. Responsiveness of the Spanish version of the "skin cancer index." J Skin Cancer. 2016;2016: 8180348 .

45. Vaidya TS, Mori S, Khoshab N, et al. Patient-reported aesthetic satisfaction following facial skin cancer surgery using the FACE-Q skin cancer module. Plast Reconstr Surg Glob Open. 2019;7(9): e2423.

46. Al-Dujaili Z, Henry M, Dorizas AS, Sadick NS. Skin cancer concerns particular to women. Int J Womens Dermatol. 2017;3(1 Suppl):S49-51.

47. Apalla Z, Lallas A, Sotiriou E, Lazaridou E, Ioannides D. Epidemiological trends in skin cancer. Dermatol Pract Concept. 2017;7(2):1-6.

48. Falk M, Faresjö A, Faresjö T. Sun exposure habits and health risk-related behaviours among individuals with previous history of skin cancer. Anticancer Res. 2013;33(2):631-8.

49. Meyer N, Pruvost-Balland C, Bourdon-Lanoy E, Maubec E, Avri MF. Awareness, knowledge and attitudes towards sun protection among skin cancer-treated patients in France. J Eur Acad Dermatol Venereol. 2007;21(4):520-5.

50. Rhee JS, Matthews BA, Neuburg M, Smith TL, Burzynski M, Nattinger AB. Quality of life and sunprotective behavior in patients with skin cancer. Arch Otolaryngol Head Neck Surg. 2004;130(2): 141-6.

51. Michielin O, van Akkooi ACJ, Ascierto PA, Dummer R, Keilholz U, ESMO Guidelines Committee. Cutaneous melanoma: ESMO Clinical Practice Guidelines for diagnosis, treatment and follow-up. Ann Oncol. 2019;30(12):1884-901.

52. Răducu L, Avino A, PurnichescuPurtan R, et al. Quality of life in patients with surgically removed skin tumors. Medicina (Kaunas). 2020;56(2):66. 
53. Bergenmar $\mathrm{M}$, Mansson-Brahme E, Hansson J, Brandberg Y. Surgical resection margins do not influence health related quality of life or emotional distress in patients with cutaneous melanoma: results of a prospective randomised trial. Scand J Plast Reconstr Surg Hand Surg. 2010;44:146-55.

54. Newton-Bishop JA, Nolan C, Turner F, et al. A quality-of-life study in high-risk (thickness $\geq$ or $2 \mathrm{~mm}$ ) cutaneous melanoma patients in a randomized trial of $1-\mathrm{cm}$ versus $3-\mathrm{cm}$ surgical excision margins. J Investig Dermatol Symp Proc. 2004;9(2): 152-9.

55. Wali GN, Gibbons E, Kelly L, Reed JR, Matin RN. Use of the Skin Cancer Quality of Life Impact Tool
(SCQOLIT) - a feasibility study in nonmelanoma skin cancer. J Eur Acad Dermatol Venereol. 2020;34(3):491-501.

56. de Troya-Martín M, Rivas-Ruiz F, Blázquez-Sánchez $\mathrm{N}$, et al. A Spanish version of the Skin Cancer Index: a questionnaire for measuring quality of life in patients with cervicofacial nonmelanoma skin cancer. Br J Dermatol. 2015;172(1):160-8.

57. Bates AS, Davis CR, Takwale A, Knepil GJ. Patientreported outcome measures in nonmelanoma skin cancer of the face: a systematic review. Br J Dermatol. 2013;168(6):1187-94. 